\title{
Pharmacoepidemiology - current opportunities and challenges
}

\author{
Jesper Hallas \\ Department of clinical pharmacology, Odense University, Winsløwsparken 19,3, DK-5000 Odense C, Denmark \\ j.hallas@dadlnet.dk
}

\begin{abstract}
Pharmacoepidemiology is defined as the the study of the use and the effects of drugs in large groups of people. It can be viewed as an epidemiological discipline with particular focus on drugs. The Scandinavians pioneered the developement of methods to study drug utilization. The ATC and DDD methodology is now a widely accepted consensus on how to categorise drugs and compare sales of drugs of different potency. The hierachy of available designs to study drug effects is described. There is an inverse relationship between the validity of a study design and its resource requirements. One of the most pervasive methodological problems of pharmacoepidemiology is the possibility of confounding by indication. Often, such confounding can only be handled by mounting a randomised trial. If a trial is unethical or unfeasible, the research question has the potential of becoming af standing controversy. The emergence of large population based prescription databases is an exciting new development in pharmacoepidemiology. The validity of drug exposure data is discussed. It is established that data from a pharmacy based database should be preferred over data from a GP based database. Whether interview data are preferable to dispensing data can not be established in general but may occasionally be inferred from the context of the research question.
\end{abstract}

\section{INTRODUCTION}

Pharmacoepidemiology can be defined as the study of the use of and the effects of drugs in large groups of people (1). Thus, it bridges the disciplines of epidemiology and clinical pharmacology. Its background is the increasing understanding that the information gathered in pre-marketing studies does not adequately describe the true health impact of a drug. First, pre-marketing trials are usually conducted in highly selected populations free of concurrent ailments or drug use, and who very poorly represent the drug users after marketing. Secondly, most drugs have important side effects that are not known at the time of marketing, either because they are too rare to be noticed in the small premarketing trials, or they are confused with unrelated, randomly occurring adverse events. One recent example of the latter is the propensity of SSRIs to cause ulcer bleeding (2). These limitations warrant the continuous post-marketing surveillance of drug dispersion in society and of drug effects, the two core elements in the definition of pharmacoepidemiology.

\section{STUDY OF DRUG USE}

Scandinavians pioneered the development of models to study drug use in populations, mainly through the work of the Drug Utilization Research Group under the auspices of WHO. Two inventions were crucial for this accomplishment (3). The first is a hierarchical system to classify drugs, the Anatomical-TherapeuticChemical (ATC) code. As an illustrative example, the ATC code for ibuprofen is shown in figure 1. It has five levels or seven digits. The first level, the M, indicates that the drug belongs to the class of drugs with action on the muskulosceletal system. The next level, indicated by 01 , specifies it further as belonging to the group of anti-inflammatory drugs, the next level A as an NSAID etc. This classification provides a consensus about how to collapse drugs with similar properties. By referring to, e.g., NSAIDs as any drug with an ATC-code of M01A in the first four digits, a researcher can avoid endless explanations of what particular drugs are the focus of a study of NSAID use. The ATC-system is not the only possible way to classify drugs, but it provides a well-elaborated, wellaccepted, reasonable consensus.

The other important development for the study of drug use in populations is the defined daily dose (DDD). It is established by an international expert panel as the typical maintenance dose for a drug when it is used by an adult for its main indication. The DDD for two different drugs should thus in principle express equipotent doses. A few illustrative examples are shown in table 1 .

The DDD should not be interpreted as a dose recommendation, but rather as a technical unit of measurement that allows us to collapse data on drugs with 
differing potency. Again, the DDD is no God-given measure and particular values may be debated. It does, however, provide a reasonable consensus. The ATCsystem and the DDDs are reviewed once annually by the WHO Collaborating Centre for Drug Statistics Methodology in Oslo, to adjust for new developments in the clinical use of the drugs.

M01AE01

\begin{tabular}{l|l|} 
Muscles/joints & M01AE01 \\
Antiinflammatoric drugs & $\left.\right|_{\text {NSAIDs }} ^{\text {Propionic acid derivates }}$ \\
Ibuprofen
\end{tabular}

Figure 1. The ATC-code of ibuprofen with an account of its elements.

Table 1. Illustrative examples of the DDD-value.

\begin{tabular}{ll}
\hline Substance & DDD \\
\hline Ibuprofene & $1.2 \mathrm{~g}$ \\
Indometacine & $0.1 \mathrm{~g}$ \\
Inhalered budesonide & $0.8 \mathrm{mg}$ \\
Inhalered terbutalin & $2.0 \mathrm{mg}$ \\
Insulin & $40 \mathrm{IE}$ \\
Levomepromazine & $300 \mathrm{mg} *$ \\
* The main indication for levomepromazine is \\
schizophrenia.
\end{tabular}

\section{Drug use statistics}

The use of systemic antibacterial antibiotics, ATCcode J01, in Norway in 1999 was 14.3 DDD per 1000 individuals per day. This common measure of drug use is known as the "therapeutic intensity". It may be construed as a surrogate measure for the pointprevalence. If all users of antibiotics took exactly one DDD per day, then the consumed amount would suffice to treat 14.3 out of 1000 persons every day. Obviously, if the actual daily intake deviates from the DDD, then the therapeutic intensity becomes a poor measure of the point prevalence.

The data on gross-volume drug use was collected through a comprehensive network of reporting from wholesale distributors. The Scandinavian model of gross volume drug statistics by use of the ATC and DDD and expressed as therapeutic intensity has gained acceptance over most of Europe. If we would appreciate its value, we may ask an American an apparently simple question like which of two states has the highest consumption of antibiotics. Without using the ATC-system or DDD, this could become a very timeconsuming exercise and would result in a fairly arbitrary answer.

\section{Individual based drug utilization statistics}

Recently, Denmark and Finland have established databases that cover the entire populations and where each prescription is recorded with the possibility of following the individual drug users prescription history. This allows for a considerable refinement of drug use statistics, by analysing drug use with the individual user as unit of analysis. For example, it is possible to estimate the prevalence, incidence and duration of use for specific drugs (4). This can be refined even further by use of techniques such as waiting-time distributions (5), Lorenz-curves (4) or analyses of composite patterns (6). By that, it is possible to screen for signs of irrational drug use.

\section{DRUGS AS CAUSES OF DISEASE}

As in most other disciplines of epidemiology, we deal with multiple causes of disease. It is almost an axiom that there are no disease entities where drug exposure is both a necessary and sufficient cause. The clinician may observe that a patient developed some unexpected adverse event after starting a drug, but he can never conclude with certainty that the drug caused the event.

This possibility of confusing adverse drug reactions with other adverse events entails two categories of problems: a delay in the recognition of true adverse drug reactions, or perceiving something as adverse drug reactions when in fact it is not. There are many examples of the former. E.g., aspirin was marketed in 1898. Gastroduodenal lesions ascribed to aspirin was not described before 1939. Not before the early 1970s, more than 70 years after marketing, could it be viewed as well documented that these lesions exist. Phenacetin was marketed in late $1890 \mathrm{~s}$, but its liability to cause renal failure was not reported before 1955 .

The other category of problems, perceiving something as adverse drug reactions when in fact it is not, is seen when old doctrines, e.g., the depressiogenic effect of betablockers, is subjected to formal testing and found to be not substantiated.

Today we have a hierarchy of available designs (table 2). The methods listed on top are less valid but also require fewer resources. Usually a suspicion arises in one of the less valid designs, for example a persuasive case report or a signal in spontaneous reporting, after which this suspicion is tested in a more valid (and resource demanding) observational design.

\section{Spontaneous reporting}

The first organised attempt to overcome the delays in recognising adverse drug reactions was to establish 
networks where clinicians could report events they suspected were adverse drug reactions, so-called spontaneous reporting schemes. The underlying rationale was that most serious adverse drug reactions occur too rarely for the individual clinician to conclude anything. For example, a GP will encounter an upper gastrointestinal bleeding caused by NSAID on the average once every third year. However, if such observations are gathered from the experience of thousands of individual clinicians, there would be a signal that would warrant further investigation.

Table 2. Hierachy of available designs in pharmacoepidemiology.

\begin{tabular}{ll}
\hline Design & Characteristic \\
\hline Case report & Hypothesis generating \\
Case series & \\
Spontaneous reporting & \\
Ecologic designs & \\
Cross-sectional design & \\
Case-control study & Hypothesis testing \\
Cohort study & \\
Cross-over designs & \\
Randomised clinical trial & Non-confounded \\
Meta-analyses & \\
\hline
\end{tabular}

The organisational framework for such spontaneous reporting originated in most western countries during the late 1960s. Since 1968, reports from a number of countries have been collected in the WHO collaborative spontaneous reporting database in Uppsala. Today it has some two million reports.

Although the spontaneous reporting schemes represented an important methodological development in the surveillance of drug safety, it has some serious limitations. First, there is a gross underreporting. A recent study in France showed that for adverse drug reactions seen in general practice only one in 25,000 is reported. Serious adverse drug reactions were reported with a frequency of one in 4,000 (7). The problem is not the underreporting in itself, but rather that it is selective and unpredictable. Thus, there are very few signals that cannot be explained by selective reporting. A second problem is that one cannot adjust for confounders. The characteristics of the drug users that gave rise to the cases are largely unknown. Even the number of exposed persons can be difficult to establish. Thirdly, the spontaneous reporting cannot reject a hypothesis, once it is raised. On the contrary, when a new controversy is covered in the medical journals or lay press, it entails a surge of reports of that particular reaction, a so-called snowball effect. Finally, the sensitivity of the method has been questioned. A review of a series of adverse drug reaction controversies showed that the spontaneous reporting was rarely crucial to the development (8).
It has been suggested that the spontaneous reporting schemes should be abandoned altogether. However, most acknowledge it as an important generator of signals that can provide the input to observational studies of a more valid design.

\section{Confounding by indication}

As stated earlier, pharmacoepidemiology can be viewed as an epidemiological discipline with drug exposure as the focus of interest. Its methodological framework is largely identical to that of other epidemiological disciplines. A researcher embarking on a pharmacoepidemiological project should go through the same considerations of sample size, selection bias, information bias, confounding, control selection and interpretation as other epidemiologists. Among the very few problems that are particular for pharmacoepidemiology is the possibility of confounding by indication.

In 1974, researchers from the Boston Collaborative Drug Surveillance Program could show that subjects who had taken aspirin had a marked reduction of their risk of having a myocardial infarction (9). This finding was not unexpected; the inhibitory effect on thrombocyte aggregation was already known. Since then, the beneficial effect of aspirin has been confirmed in numerous randomised trials.

If a researcher would mindlessly repeat the BCSDP study today, he would come to the opposite conclusion; that users of aspirin have a higher incidence of myocardial infarction than others. Today, aspirin is rarely prescribed as an analgesic. Rather, it is selectively prescribed to high-risk persons with an arteriosclerotic disposition. Aspirin lowers their risk of arteriosclerotic events such as myocardial infarction, but not enough to compensate for their elevated baseline risk. Obviously, this is a confounder problem. The exposure, aspirin, is coupled to a strong predictor of the studied outcome, myocardial infarction. An important question now is whether it is possible to adjust for confounding by indication by conventional means in an observational design.

This was the issue in an elegant study by Miettinen (10). The objective was to study the association between use of oral coumarins and development of venous thromboses. He used data from the BCSDP in a case-control design. An analysis with adjustment for age and gender showed an odds ratio of 27. After adjustment for known predisposition for venous thrombosis, the odds ratio fell to 4 . Oral coumarins protect against venous thrombosis. Thus, the true OR as it would have appeared in a large, well-conducted randomised trial, should be below one. There are two important conclusions; first, confounding by indication is a very strong confounder, in this case shifting the odds ratio from below one to 27 . Second, even a wholehearted attempt to adjust for this confounder left a strong residual confounding, since the odds ratio 
would not go below 4 . The consequence is aptly expressed in the title, "The need for randomization in the study of intended drug effects".

Often it would be impossible to mount a randomised trial, for reasons of ethics or resource requirements. If it is an issue with a strong confounding by indication, it would have the potential of becoming a standing controversy, in which consensus cannot be reached. A good example is the controversial association between SSRIs use and suicides (11). A number of studies have shown higher incidence of suicides among users of SSRIs compared to users of tricyclic antidepressants. Whether this reflects a true difference in effect or subtle differences between the users is as yet unresolved. The SSRIs have a much lower acute toxicity than the tricyclic antidepressants, which might be a good reason to prescribe these selectively to the patients with the strongest suicidal impulses (12). Such differences between users could be very subtle and difficult to capture in an observational study. A similar recent controversy was the finding of a higher cardiovascular mortality among users of calcium blockers compared to other antihypertensives (13). Although the authors were conscious about the possibility of confounding by indication and made every effort to adjust for it, we cannot rule out that the finding is explained by subtle differences in the characteristics of users of antihypertensives. In clinical trials, the treatments are allocated by randomisation, and the subjects in each treatment arm are therefore in principle comparable, even for unmeasured or unknown confounders.

\section{PHARMACOEPIDEMIOLOGIC DATABASES}

One of the main methodological developments in pharmacoepidemiology has been the emergence of large databases with more or less complete capture of individual drug use and clinical outcomes for large populations. It makes it possible to gather detailed systematic information on large groups of drug users and to link it to information concerning suspected adverse outcomes in individual users. With few exceptions, they are all derived from administrative systems designed to proces financial refund, e.g. as in health maintenance organisation.

The advantages of using pharmacoepidemiologic databases in research are:

1. Data are free of recall or interviewer bias.

2. Data are already recorded when the controversy arises. It is possible to conduct studies on a historic data set and provide an answer quickly. Also, if a suspected adverse drug effect becomes controversial, it may be difficult for subsequent researchers to study the subject. The issue becomes "polluted", e.g., with diagnostic suspicion bias. One way to overcome this is to use a historic data set from before the adverse drug reaction was suspected.

3. Costs are usually low.
The most important limitations of pharmacepidemiologic databases are:

1. They are not suitable for the study of drug effects where the timing of drug intake is critical, i.e., short-term hyperacute effects.

2. It may be uncertain whether the patient has actually ingested the drug.

3. Cases where the endpoint is poorly described by the coding system.

4. They do not cover medication bought over the counter.

The most important pharmacoepidemiologic database in the present scientific scenario is undoubtedly the British General Practitioners Research Database (GPRD) which is based on administrative systems for general pratice. It has provided detailed coverage of diagnoses, prescriptions and certain other variables such as smoking status and weight for some four million persons in UK since 1990 (14). Diagnoses are recorded by a GP-specific coding system, the OXMIS. Validation studies have shown that $90 \%$ of specialist referrals and hospital discharge notes can be found in the database. The GPRD is owned by the NIH, but it is possible for researchers in other settings and countries to buy data sets from the GPRD.

\section{Quality of exposure data}

It is a standing issue which measure of drug exposure that best covers the patients' actual ingestion of drugs. There are essentially three candidates: Data may be found from a GP's administrative system as in the GPRD, from a database based on prescription refund claims, or from patient interview. The relationship between these sources is described in figure 2 .

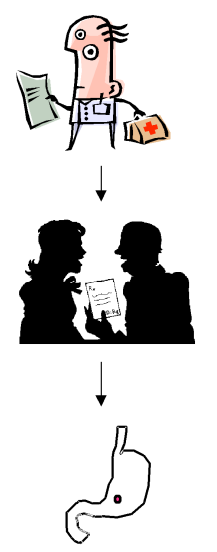

Physician administrative system, e.g. GPRD

Pharmacy-based prescription database

Interview

Figure 2. The process from prescribing to ingesting medication and its equivalent data sources.

There are several important uncertainties in the flow chart. First, the physician may issue a prescription, but the patient may not redeem it at the pharmacy. Secondly, the patient may redeem it at the pharmacy, 
but he may not take the drug. Thirdly, he may take the drug, but fail to report so when interviewed about it. Or conversely, he may report to take a drug, when in fact he does not. In addition, there are other potential uncertainties, i.e. some technical malfunctions may impair the transfer of data from GPs administrative system or from the pharmacy records. These last uncertainties are usually unimportant.

The desired information is placed in the core of the diagram, namely what actually passes the patient's stomach. Unfortunately, this is highly untangiable and can only be studied directly under very artificial circumstances. The first major uncertainty, i.e., the differences between what has been prescribed at the doctor's office and what has been dispensed at the pharmacy is termed primary non-compliance. It has been studied in the setting of the Scottish research database MEMO, which is based on prescriptions redeemed at the pharmacy. A large rural practice recorded all prescriptions during a three month period (15). The prescriptions were subsequently retrieved in the MEMO database. Most drug classes showed a deficit of $3-8 \%$. For oral contraceptives, $25 \%$ of the prescriptions were not redeemed! The authors concluded that drug exposure should be assessed from pharmacy records rather than GP prescription records.

The other uncertainties are much more difficult to study. Obviously, the patient may not take the medication that has been dispensed at the pharmacy, which might lead us to think it was better to ask the patient than to use pharmacy records. However, as any clinician would know, the average patient is often surprisingly ignorant about what medication he is supposed to take every day, and his account of drug use may be highly unreliable. The discussion about which data source, interview or prescription databases, best represents the actual drug intake cannot be resolved since there is no way to obtain the correct answer. There are a number of studies on relationships between interview and pharmacy data, but these are at best only studies of agreement, not of validity.

Sometimes the best choice of data source may be inferred from the context. For example, it has been shown recently that users of antibiotics with effect against Chlamydia Pneumonia have a lower incidence of myocardial infarction than users of other antibiotics during the past three years (16). This study was performed by use of a prescription database, which was probably the best choice. Very few real-life patients can give a reliable account of their antibiotic use during the past three years - in sufficient detail to allow a distinction between antibiotics with and without effect against Chlamydia Pneumoniae.

On the other hand, the databases cannot give information about exact timing of drug intake. For example, a recent controversy is whether sildenafil causes myocardial infarction (17). Obviously, intake of sildenafil is closely correlated to having sex, which by itself has a weak infarction provoking effect (18). Thus, a formal study of whether sildenafil causes myocardial infarction would require detailed information about the exact timing of the triad of intake of sildenafil, sexual activity and myocardial infarction. No database could provide this.

\section{SUMMARY AND CONCLUSIONS}

Pharmacoepidemiology is the study of the use of and the effects of drugs in large groups of people. It has most of its methodological framework in common with other disciplines of epidemiology. Among its few particularities is the possibility of confounding by indication, which may be impossible to solve in an observational design. There are worldwide a number of databases with comprehensive registration of drug use and outcomes for entire populations. These are, when used judiciously, an important tool in the study of unknown or controversial drug effects.

\section{REFERENCES}

1. Strom BL. What is pharmacoepidemiology? In Strom BL (ed): Pharmacoepidemiology, $2^{\text {nd }}$ edn. Chichester: John Wiley \& Sons, 1994: p 3.

2. de Abajo FJ, Garcia Rodriguez LA, Montero D. Association between selective serotonin reuptake inhibitors and upper gastrointestinal bleeding: a population-based case-control study. Br Med J 1999; 319: 1106-9.

3. Lee D, Bergmann U. Studies of drug utilization. In Strom BL (ed): Pharmacoepidemiology, $2^{\text {nd }}$ edn. Chichester: John Wiley \& Sons, 1994: p 379.

4. Hallas J, Nissen A. Individualised drug utilization statistics. Analysing a population's drug use from a perspective of individual users. Eur J Clin Pharmacol 1994; 47: 367-72.

5. Hallas J, Gaist D, Bjerrum L. The waiting-time distribution for prescription drugs. Epidemiology 1997; 8: 666-70.

6. Gaist D, Hallas J, Hansen NCG, Gram LF. Are young adults with asthma treated sufficiently with inhaled steroids? A population-based study of prescription data from 1991 and 1994. Br J Clin Pharmacol 1996; 41: 285-9. 
7. Moride Y, Haramburu F, Requejo AA, Begaud B. Under-reporting of adverse drug reactions in general practice. Br J Clin Pharmacol 1997; 43: 177-81.

8. Venning GR. Identification of adverse reactions to new drugs II. Br Med J 1983; 286: 289-92.

9. Boston Collaborative Drug Surveillance Program. Regular aspirin intake and acute myocardial infarction. $\mathrm{Br}$ Med J 1974; 1: 440-4.

10. Miettinen OS. The need for randomization in the study of intended effects. Stat Med 1983; 2: 267-71.

11. Teicher MH, Glod C, Cole JO. Emergence of intense suicidal preoccupation during fluoxetine treatment. Am J Psychiatry 1990; 147: 207-10.

12. Isacson G, Redfors I, Wasserman D, Bergman U. Choice of antidepressants: questionnaire survey of psychiatrist and general practitioners in two areas of Sweden. Br Med J 1994; 309: 1546-9.

13. Psaty BM, Heckbert SR, Koepsell TD, Siscovick DS, Raghunathan TE, Weiss NS, et al. The risk of myocardial infarction associated with antihypertensive therapies. JAMA 1995; 274: 620-5.

14. Garcia Rodriguez LA, Perez Gutthann S. Use of the UK General Practice Research Database for pharmacoepidemiology. Br J Clin Pharmacol 1998; 45: 419-25.

15. Beardon PHG, McGilchrist MM, McKendrick AD, McDevitt DG, MacDonald TM. Primary non-compliance with prescribed medication in primary care. Br Med $J$ 1993; 307: 846-8.

16. Meier CR, Derby LE, Jick SS, Vasilakis C, Jick H. Antibiotics and risk of subsequent first-time acute myocardial infarction. JAMA 1999; 281: 427-31.

17. Feenstra J, van Drie-Pierik RJ, Lacle CF, Stricker BH. Acute myocardial infarction associated with sildenafil. Lancet 1998; 352: 957-8.

18. Muller JE, Mittleman MA, Maclure M, Sherwood JB, Tofler GH. Triggering myocardial infarction by sexual activity. Low absolute risk and prevention by regular physical exertion. JAMA 1996; 275: 1405-9. 